\title{
Pathological Sit-To-Stand Models for Control of a Rehabilitation Robotic Device
}

\author{
Ludovic Saint-Bauzel ${ }^{1}$, Viviane Pasqui ${ }^{1}$, Bruno Gas ${ }^{2}$, Jean-Luc Zarader ${ }^{2}$ \\ ${ }^{1}$ Université Pierre et Marie Curie-Paris6, \\ FRE-2507 Laboratoire de Robotique de Paris (LRP), \\ Fontenay-aux-Roses, F-92265 France; \\ saintbauzelerobot.jussieu.fr, pasqui@robot.jussieu.fr \\ ${ }^{2}$ Université Pierre et Marie Curie-Paris6, \\ EA2385 Groupe Perception et Réseaux Connexionniste (PRC), \\ Ivry sur Seine, F-94200 France; \\ gas@ccr.jussieu.fr, zarader@ccr.jussieu.fr
}

\begin{abstract}
The aim of the work presented in this paper is to realise the model for the control of a robotic interface for equilibrium assistance during sit-to-stand transfer.

Here, it is thought that interactive robotic devices, as human-centered robotics, is more comfortable and more efficient that traditionnal technical devices. One supposes the need of virtual model of the pathology. This model, called observer, aims at being used in the smoothing control part of this assisting device. A usefull property of this observer should be a prediction ability of a postural instability.

This article presents a study of different neural solutions : a Neural Trajectory Generator and a Neural Predictive Observer (NPO). Records used for the learning have been done from healthy people that stand up normally and quickly. Some tests will also be done in patients with cerebellar syndrome disease.

The presented experimental results show the good accuracy of these approaches whatever the speed of the movement.
\end{abstract}

\section{Introduction}

This work concerns the control of robotic devices interacting with patients during rehabilitation exercises. The interaction between the assisting device and the patient can be considered as a control loop (cf. fig. 1). In this case, the patient is assimilated to an unknown system and the robot to an actuator regulating disor- ders. The stabilisation is obtained comparing the patient's state with a physiological trajectory generation [6]. Our aim is to improve the human-robot interaction. Some classical approach to improve a control is to include a model in the control loop.

This article will focus on the design of models for STS trajectories in joints space of both diseased and healthy subjects. The different models will have different input vector $(i)$ but they always return computed joint trajectory $\left(q^{*}\right)$ (cf. fig. 1).

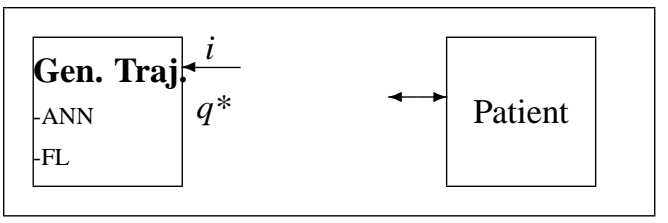

\section{Figure 1. Control system overview Attention image à réparer!!!!}

The particular device studied is a robot [2] helping for STS (cf. fig 2). Composed of two degrees of freedom independent handles on an active mobile platform, this robot can interact with a person during the STS motion and also all along the walk.

The kind of control we wanted to evaluate on this device concerns only the sagittal movements that is the reason why we will reduce the Sit-To-Stand(STS) modelisation to a 2 dimensional problem (fig. 8). We suppose that the latteral motion are reduced by the handle grasping.

The STS transfer(fig. 8) is a complex motion that 


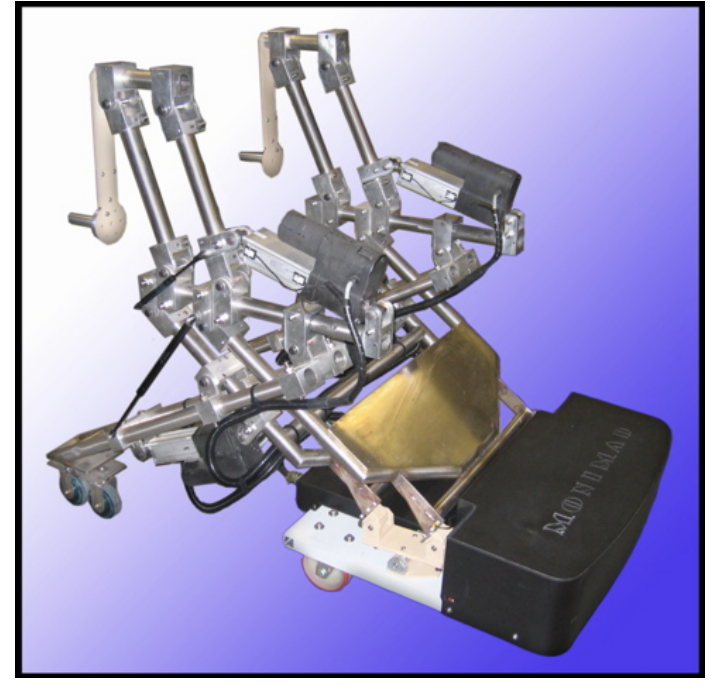

Figure 2. Assistive device: The robotic interface MONIMAD

combines the will, the sensori-motor action with a fine regulation all along the process. Diseases that affect this movement are very often pathologies concerning the regulation which is usually considered as the cerebellum role.

One of the main problems that must be solved to implement a model based controller depicted in fig. 1 is that there is not any suitable model for healthy and/or cerebellar disease movement. Focused on STS trajectories, the models presented in this article are used to control an assistive device during a STS transfer for diseased subjects. An Artificial Neural Network (ANN) based modelling are used, which structure has been designed as an intermediate solution between complex models developped in neuroscience [7], [8], [9] and simplistic solutions often used in robot control [10], [11]. As a result, the proposed models will be studied for the following properties :

- Generalization : the model performs equally for normal speed STS as for fast one.

- Specialization : the solution must be able to fit an individual disease expression.

- Disease ability : the best solution will be tested on diseased trajectory.

\section{Method}

\subsection{Artificial Neural Networks}

2.1.1. Description. ANN are commonly used in literature dedicated to problems involving some behaviours that are not polynomials and must be learned. This software structure (fig. 3) is composed of many artificial neurons. It is organized in layers. The first layer is directly connected with the inputs of the system, the last layer represents the outputs of this structure and the layers between are called hidden layers.

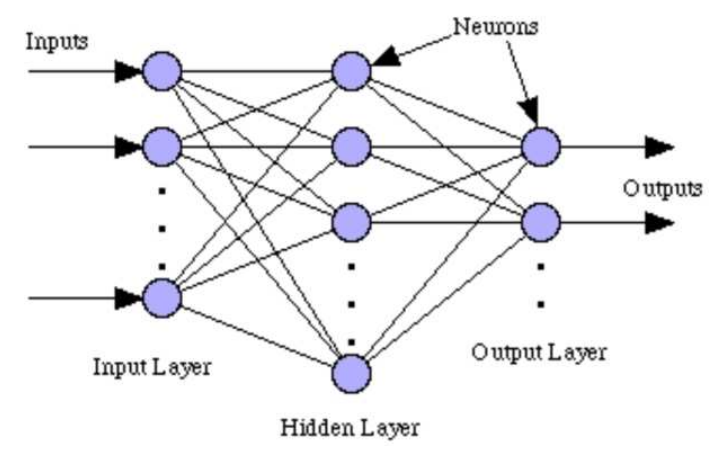

Figure 3. Artificial Neural Network architecture

The artificial neuron is a computer implementation of the neuron behaviour. Indeed a neuron is an integrator of signals, and the result is an activation of the neuron which is transmitted on the axon. The artificial one (fig.4) is composed of weighted inputs( $w)$, an integrator which is a sum $\left(\sum\right)$ and finally an activation function $(f)$ computing the output (axon).

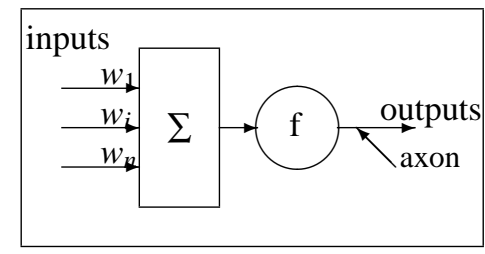

Figure 4. One artificial neuron

2.1.2. Non-linearity. One of the motivation of this choice is the ability of this method to work through some kind of non-linearity. Simple examples of nonlinearity are movement discontinuities : when a person pulls up a feet, or when he falls. Others kinds of discontinuities are singularities in the kinematics model. 
These singularities can be found in some particular configurations. Therefore, it is important to choose a method that deals with these problems.

In a classical mechanical approach, the trajectories of a system are considered as polynomial functions. It is often considered as a good approximation. ANN are able to generate non-polynomial solutions. It could be interesting to see if this property can be an asset for the solution accuracy.

2.1.3. Learning. The flexibility is defined as system ability to perform particular problems without changing its structure. In the proposed solutions, flexibility is one of the most important thing that leads the choice of learning based approach. As it is mentioned in the introduction part, a particularity of the gait and STS diseases, is that they require a solution adapted to each person. ANN seems to be the best way to achieve this goal. In the studied cases, the activation functions will be hyperbolic tangent $f$ defined in equation (1).

$$
f(x)=\tanh (x)=\frac{e^{x}-e^{-x}}{e^{x}+e^{-x}}
$$

The approach in the edge of neuroscience and robotic motivates the choice of a classical learning approach in ANN domain based on back-propagation [17], [18]. This learning method is also based on the relation (2):

$$
g=\sum\left(w_{i} \cdot g_{i}\right)
$$

where $g$ is a vector of precedent layer outputs $\left(g_{i}\right)$ and $w_{i}$ are the weights of the links and $i$ is the number of the current layer. The weights learning $\left(w_{i}\right)$ are based on a gradient backpropagation expressed as the expessons 3 in

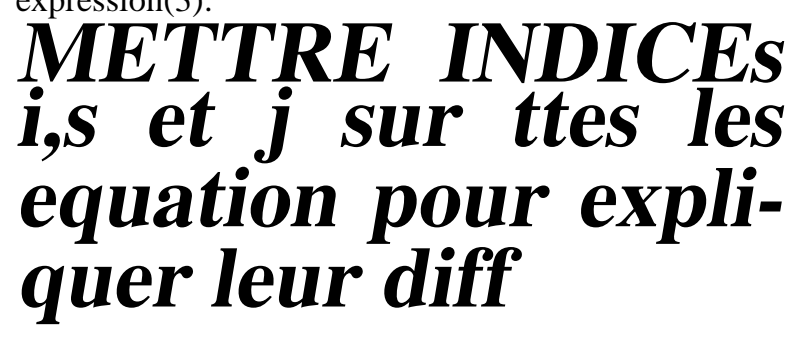

$$
w_{s+1}=w_{s}-\alpha \cdot L G_{s}
$$

with $s$ as learning step, $\alpha$ as learning rate and $L G_{s}$ as local gradient.

Local gradient is computed with the backpropagation algorithm and the computation basis in each step $\mathrm{k}$ is a cost function gradient $G_{s}$ expressed for the $j^{\text {th }}$ output. The back propagation learning tries to minimize the Neural Network (NN) objective function $(f c)$. This function is a simple Root Mean Square Error between the desired trajectory $\left(q_{j}\right)$ and the NN outputs $\left(q_{j}^{*}\right)$.
2.1.4. Motivation. According to the literature, an ANN is able to fit the considered problem of prediction. But there are few works dealing with the ANN dimensions. In this lack of information case, problems like overfitting can occur. Indeed the work presented in [20] describes a method to generate FES patterns but the obtained structure is not studied. So the understanding of how and why the ANN achieves the problem is there very difficult. That's the reason why this paper puts the stress on the results accuracy and in the description of the ANN.

\subsection{Neural Trajectory Generator}

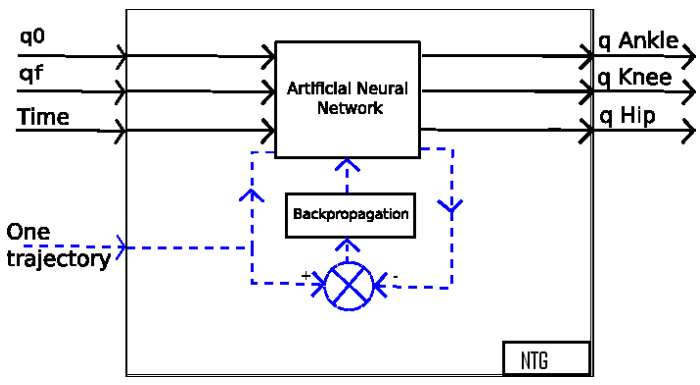

Figure 5. NTG System Description Attention image changée!!!!

2.2.1. Description. As noticed in the figure 5 , the Neural Trajectory Generator (NTG) takes as input the initial angular values $\left(q_{0}\right)$, the final angular values $\left(q_{f}\right)$ and a time vector which is used as time reference for the movement. The outputs will be angulars trajectories values. The initials values will be particularly different, indeed for the learning process it is necessary to give some angular values, in this paper we only give one set of STS trajectories. This initial values and also the initial process which is the learning of the neural network are represented with a dashed line.

2.2.2. Parameters. Following an evaluation of different NN sizes, the best NN obtained was composed by 5 hidden cells. This NN is structured as one layer perceptron with hyperbolic tangent (see relation (1)) as activation functions.

\subsection{Neural Predictive Observer}

2.3.1. Description. As noticed in figure 6, the only difference between inputs and outputs is the time reference. Indeed the outputs are angular forward values of the inputs. For the learning process the database is a set of angular values of one kind of STS: normal and fast. This initial values are used in the initial process 
which is the learning of the neural network represented on figure 6 with a dashed line.

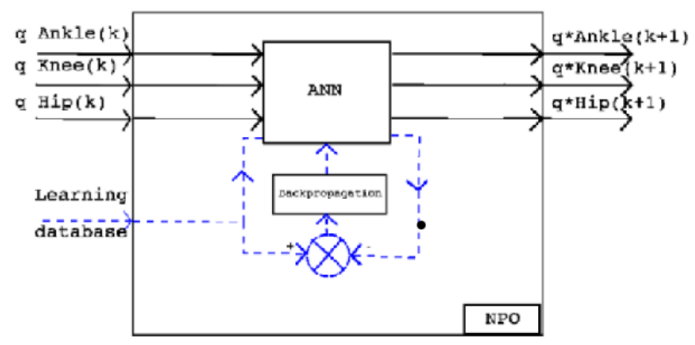

Figure 6. NPO System Description

The NPO can be represented as in figure 6, $\mathrm{q}(\mathrm{k})$ is a vector composed of the three angular values (ankle, hip and knee values) at instant $\mathrm{t}$ and boxes named $\mathrm{d}$ are delay functions (je ne vois nulle part de box "d": à expliquer.

The NN inputs size is $\mathrm{q}(\mathrm{k})$ size multiplied by sliding window size : 3xBW (Backward Window is a scalar value defining the time sliding window size). For each input in one STS trajectory learning, only 3 new values are given. Others values are obtained from the last inputs with $d$. This NN provides in each step the three angular values in forward time : $\mathrm{q}^{*}(\mathrm{k}+1)$.

So, we have a prediction on the angular values there are used in the postural state.

\subsection{Linear Predictor (LP)}

As we know, there is no predictive approach for human STS movement. So we describe a Linear Predictor (LP) in order to have a comparison point. This LP is a simple linear extrapolation. The aim of a linear extrapolation is to define the line two parameters, $a$ and $b$ in relation (4):

$$
y_{k}=a \cdot k+b
$$

This is obtained with a pseudo-inverse (noted with sym$\mathrm{bol}^{+}$) of the BW last parameters as shown in expressions (5):

$$
\left\{\begin{array}{c}
u_{k}=\left[\begin{array}{ll}
k & 1
\end{array}\right]^{T} \\
D_{k}=\left[\begin{array}{lll}
u_{k}-B W & \ldots & u_{k}
\end{array}\right] \\
Y_{k}=\left[\begin{array}{lll}
y_{k}-B W & \ldots & y_{k}
\end{array}\right] \\
D_{k}^{+}=D_{k}^{T} \cdot\left(D_{k} \cdot D_{k}^{T}\right)^{-1} \\
A B=Y_{k} \cdot D_{k}^{+}=\left[\begin{array}{ll}
a & b
\end{array}\right] \\
y_{k+1}=A B \cdot u_{k+1}
\end{array}\right.
$$

\section{UNE CONCLUSION S'IMPOSE!!!!!!!!!!!!!!!!}

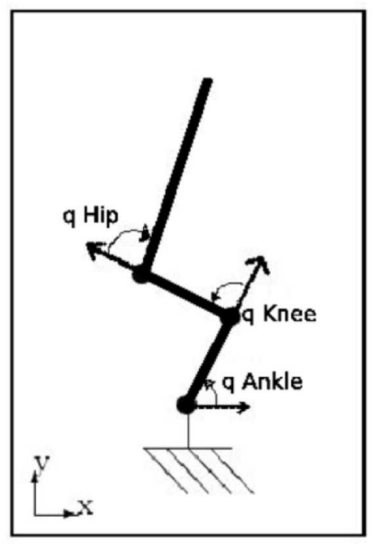

Figure 7. A three links model

\section{Application}

\subsection{Biomechanical model}

From the 3-bar mechanism (fig. 7), the dynamical model is determined according to classical equations (eq.6), $q(t)$ represents the vector (3X1) of the 3 angular values, $H(q)$ is the $3 \mathrm{X} 3$ mass matrix , $h(q, \dot{q})$ is the vector (3X1) of Coriolis and rotation effects and $C(q(t))$ is a vector of gravity terms.

$$
\begin{array}{r}
H(q) \ddot{q}+h(q, \dot{q})+C(q(t))=\tau+J^{T} \lambda \\
q_{\min } \leq q \leq q_{\max } \\
q\left(t_{0}\right)=q 0, q\left(t_{F}\right)=q_{F} \\
\dot{q}\left(t_{0}\right)=q 0, \dot{q}\left(t_{F}\right)=0
\end{array}
$$

As far as it is not a redundant system, there is no need of Lagrangian coefficient : $J^{T} \lambda=0$

\subsection{Data acquisition}

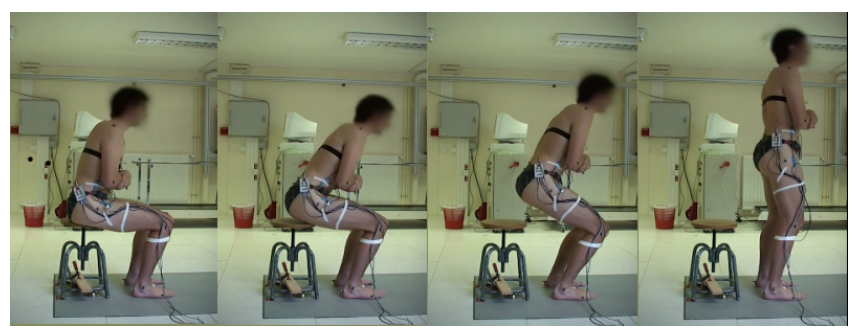

Figure 8. A Sit-To-Stand movement

Records (fig.8) on healthy persons are performed with goniometers placed on ankle, knee and hip of the right leg. Statistical informations concerning the goniometers are presented in table 1, where SD is the Stan- 
Table 1. Statistical information of goniometers

\begin{tabular}{|c||c|c|}
\hline & $\begin{array}{c}\text { SD } \\
(\operatorname{rad~x~10-2})[\mathrm{rel} .(\%)]\end{array}$ & $\begin{array}{c}\text { Max } \\
\left(\mathrm{rad} \times 10^{-2}\right)[\mathrm{rel} .(\%)]\end{array}$ \\
\hline hip & $0.32[1.17]$ & $0.54[1.96]$ \\
knee & $0.18[0.16]$ & $0.50[0.45]$ \\
ankle & $0.20[0.09]$ & $0.01[0.23]$ \\
\hline
\end{tabular}

dard Deviation and Max represents the maximum error. The persons were asked to stand-up normally with crossed arms on chest. They were also asked to standup as quickest as they can.

Concerning the diseased people, records has been done with a motion capture system developped by Motion Analysis. This system give the position of some points. So a geometrical model is used to reconstruct the angular values. This reconstruction can cause some discontinuities, so we have to be aware of these problems in the results interpretation.

\subsection{Specialisation}

In this paper, it is considered that if the observer keep a good accuracy in normal speed and in fast speed, then it is able to keep it all along the speeds between these two extrema. It has been decided to look at the speed of the movements as a proof of specialisation of this approach. Using NPO or NTG should be interesting only if this method may be specialized for one person and if it is able to have a good accuracy in all the situations used.

\subsection{Learning process}

For these tests, learning for NTG and NPO is stopped with one verticalisation record results which is used as a validation database. The learning database is composed of movements from one person in normal speed (9 records) and in fast speed (11 records).

First, the results will be studied in the same speed field of data. Then, a cross test will be performed in order to know if a network which learns a normal (resp. fast) STS trajectory can observe a fast (resp. normal) movement.

Indeed this solution should be able to identify a STS for every speed.
Table 2. Comparaison between polynomial optimization and neural network approach

\begin{tabular}{|c||c|c|}
\hline & NTG (rel) & KUZE(rel) \\
\hline A & $0.0389(1.78)$ & $0.0028(0.81)$ \\
B & $0.0207(0.95)$ & $0.0376(1.72)$ \\
C & $0.0160(0.73)$ & $0.0150(0.69)$ \\
D & $0.0159(0.73)$ & $0.0100(0.46)$ \\
\hline
\end{tabular}

where $N N$ is a neural network with one hidden layer composed of 5 cells.

\section{Results}

\subsection{Preamble}

Some first evaluation tests has been done on a small database of STS of four different subjects (A, B, C, D). These tests may answer the questions :

- "Can a neural observer learns a human movement?" - "Is the non-polynomial property an asset for the problem?"

The comparison is made between KUZE method [19] and NTG describes below. The table 2 presents the RMSE JAMAIS VU AVANT:EXPLIQUER of these two methods along the STS motions. The learning of the NTG is made with only A data. As far as the learning database is very small wich is not the best conditions for the NTG we can see comparable results.

So a neural solution is able to learn a human with almost same accuracy than a polynomial approach.

fig. 9 presents a curve describing how the nonpolynomial approach (dashed line called $\mathrm{NN}$ ) can reach a better accuracy than polynomial one (above line called KUZE).

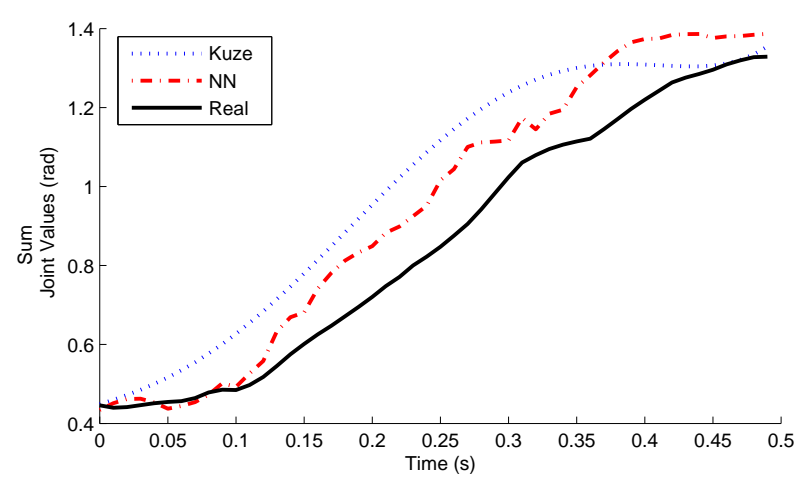

Figure 9. Joint trajectories comparison for STS of subject $B$ 
Table 3. RMSE and MAE of the best neural network for two speed of STS

\begin{tabular}{|c||c|c||c|c|}
\hline KUnits & $\begin{array}{c}\text { KUE } \\
\operatorname{rad}(\%)\end{array}$ & $\begin{array}{c}\text { NTG } \\
\operatorname{rad}(\%)\end{array}$ & $\begin{array}{c}\text { LP } \\
\operatorname{rad}(\%)\end{array}$ & $\begin{array}{c}\text { NPO } \\
\operatorname{rad}(\%)\end{array}$ \\
\hline Normal & $0,0164(0.75)$ & $0,0229(1.05)$ & $0.0001(0.0041)$ & $0.0037(0.159)$ \\
Fast & & & $0.0001(0.0041)$ & $0.0030(0.128)$ \\
Fast to Slow & & & $0.0001(0.0041)$ & $0.0024(0.099)$ \\
Slow to Fast & & & $0.0001(0.0041)$ & $0.0021(0.0859)$ \\
\hline
\end{tabular}

\subsection{Generalization}

This section puts the light on a comparison between KUZE, LP, NTG and NPO in the generalization point of view. In this paper, a method is considered with ability to generalization when it can keep a comparable accuracy when it is tested on another speed database. Figure 10 presents outputs of NPO learned on fast speed applied to normal speed data, it is interesting to notice the similarities between NPO outputs and real values whereas the learning is done on another speed database. LP is also performing good results in the different speed databases.

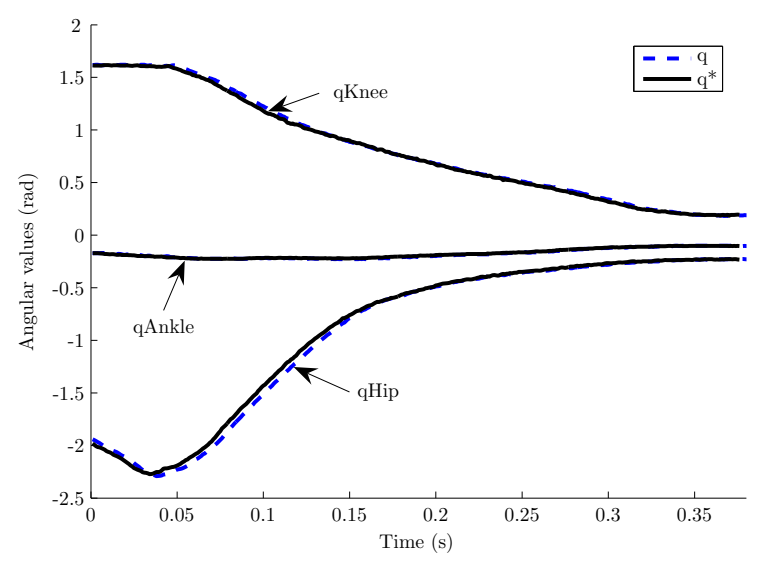

Figure 10. Real angular trajectories and neural network output for the 6th normal STS

\subsection{Specialization}

In order, to show the specialisation of this approach the best NPO learned with data of person A has been evaluated on four STS records of person B. As presented in table 4, it can be noticed that the root mean square output errors are ten time upper than the learned one. It is very interesting because we can say that the NPO solution has learned only one person STS behaviour with good accuracy. The NPO is looking for a solution defining a virtual model of this person.
The general definition of LP doesn't allow any specialisation. As far as it is defined as a minimum error straight line that fit the precedent values. So there is no parameter defined that concern individual motion expression.

Table 4. Results on STS trajectories of other person

\begin{tabular}{|c|c|c|c|c|}
\hline Num & 1 & 2 & 3 & 4 \\
\hline NPO & 0.0383 & 0.0299 & 0.0282 & 0.0177 \\
LP & 0.0001 & 0.0001 & 0.0001 & 0.0001 \\
\hline
\end{tabular}

\subsection{Disease Ability}

In our point of view the best neural based solution is the NPO. Because of its accuracy, specialization and ability in data reduction, we decided to evaluate this neural structure on diseased patient. In the table 5 and in the figure 11, we can notice that this structure is suitable for diseased motion. It is remarkable to see that LP gives also very good results on this pathological trajectory.

Table 5. RMSE Results for NPO and LP with patient 2 and 4

\begin{tabular}{|c|c|c|}
\hline & Patient2 & Patient 4 \\
NPO & 0.0023 & 0.0025 \\
LP & 0.0001 & 0.0001 \\
\hline
\end{tabular}

\section{Conclusion}

In this paper, we present two modelisation methods:

- trajectory generation approach

- prediction

In the trajectory generation we can compare KUZE and NTG methods. It appears that even if NTG are 


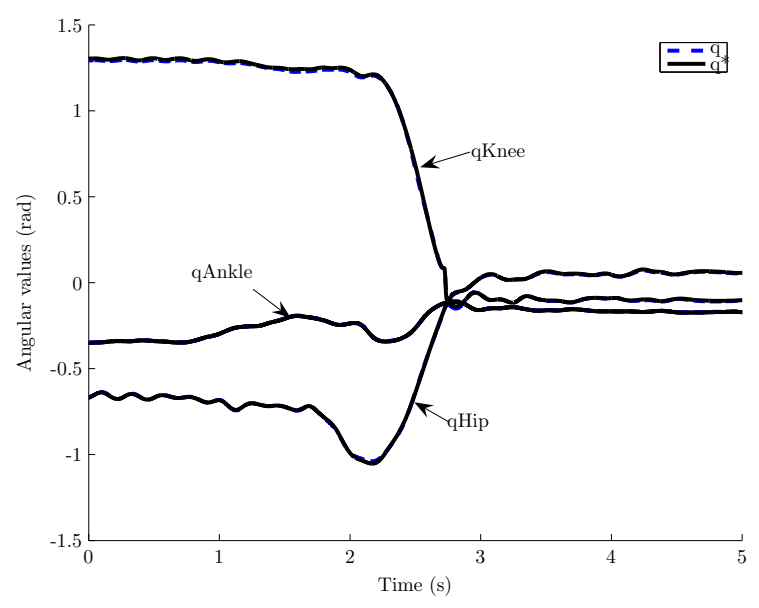

Figure 11. Real angular trajectories and neural network output for cerebellar patient4

lesser???? than KUZE method, it is interesting to say that the results obtained have the same size. It has shown that a neural approach can fit the problem. In addition, we studied 2 different prediction approaches: neural and linear. Specialisation property excepted, the LP seems to be the best approach for the frequency used $(100 \mathrm{~Hz})$. With as an asset the fact that we don't need any data to define this prediction model. As far as we consider that we want this structure to be used in rehabilitation devices, we need an approach that it is a representation of the pathology. Because the models aim also to be used as an evaluation system. According to this critical property the NPO is usefull to be used in a model based control of the rehabilitation robotics. One of the ANN properties that we didn't develop in this paper is parcimonial abilities. That means we can develop some strategies to minimize the number of input which are for the moment 3 angular values. Reducing this number could simplify the rehabilitation process. Indeed, the least sensor number we take on the subjects gives the simpliest and the most applicable process in real applications. This is the main reason why we will continue with the Neural Based Approach. Following this realist approach, we can notice that many learning results obtained are done with less than ten STS transfers and need only some minutes to be computed (10$20 \mathrm{mn}$ ). This is another positive argument to consider that a neural based approach is realistic to be used in the control of an assistive device.

\section{References}

[1] R. Reiner and L. Lunenburger and G. Colombo, humancentered robotics applied to gait training and assement., Journal of rehabilitation research and development, Vol 43,
N 5, pp 679-693, 2006.

[2] P. Médéric and V. Pasqui and F. Plumet and P. Bidaud and J.C. Guinot, Design of a Walking-aid and Sit-to-Stand Transfer Assisting Device for Elderly People, 7th Int. Conference on Climbing on Walking Robots (CLAWAR'04), 2004

[3] Fukuda T., Kubota N., Computational Intelligence for the Robotics and Automation, ISIE'99

[4] Nagai K., Nakanishi I. and Hanafusa H. Assistance of Self-Transfer of Patients Using a Power-Assisting Device, International Conference on Robotics and Automation ,Taipei, 2003

[5] Kamnik R, Bajd T., Robot assisted standing-up., In: Proceedings of the IEEE International Conference on Robotics and Automation, 24-28 April 2000. San Francisco, CA. p. 2907-12.

[6] V. Pasqui and P. Bidaud, Bio-mimetic trajectory generation for guided arm movement during assisted sit-to-stand transfer. Proc. 9th International Conference on Climbing and Walking Robots, Bruxel, Belgium, 2006.

[7] Manto, M., Godaux, E., Jacquy, J., Cerebellar hypermetria is larger when the inertial load is artificially increased . Ann. Neurol. 35: 45-52, 1994.

[8] Albus J. S., Theoretical and experimental aspects of a cerebellar model. $\mathrm{PhD}$. Dissertation, University of Maryland, 1972.

[9] Schweighofer N., Arbib M., Dominey P. A model of the cerebellum in adaptive-control of saccadic gain 1: the model and its biological substrate, Biol. Cybernet. 75 (1) 19-28, 1996.

[10] Kuo A.D. An optimal control model for analyzing human postural balance. IEEE Trans Biomed Eng 42:87101, 1995.

[11] P. Sardain and G. Bessonnet, Forces Acting on a Biped Robot Center Of Pressure-ZMP IEEE tran Syst., Man., Cybern. A, vol. 34, sept. 2004

[12] Martin BJ, Bobrow JE. Minimum effort motions for open chain manipulators with task-dependent end effector constraints. Int. J. Robot. Res., 8:213-24, 1999.

[13] A. Lapedes and R. Farber, Non-linear signal prediction using neural networks : Prediction and system modeling, Los Alamos National laboratory report, LA-UR-87-2662, 1987

[14] B. Gas, J.L. Zarader, C. Chavy (2001), A New Approach to Speech Coding : The Neural Predictive Coding, Journal of Advanced Computational Intelligence, Vol. 4, pp.120127.

[15] Uno Y, Kawato M, Suzuki R. Formation and control of optimal trajectory in human multijoint arm movement: minimum torque-change model. Bio Cybern 1989.

[16] M. Kawato, Y. Maeda, Y. Uno and R. Suzuki Trajectory formation of arm movement by cascade neural network model based on minimum torque-change criterion, Biological Cybernetics - 2004

[17] Hagan, M. T., and M. Menhaj, Training feedforward networks with the Marquardt algorithm, IEEE Transactions on Neural Networks, vol. 5, no. 6, pp. 989-993, 1994.

[18] M. T. Hagan, H. B. Demuth, and M. H. Beale, Neural 
Network Design, Boston, MA: PWS Publishing, 1996.

[19] Kuzelicki, Synthesis of standing-up trajectories using dynamic optimization, gait and posture,2005

[20] Kamnick, Nonlinear Modeling of FES-Supported Standing-Up in Paraplegia for Selection of Feedback Sensors DCS Tech Report, 2005 